\title{
BMJ Open Psychoeducational and rehabilitative intervention to manage cancer cachexia (PRICC) for patients and their caregivers: protocol for a single-arm feasibility trial
}

To cite: Buonaccorso L, Bertocchi E, Autelitano C, et al. Psychoeducational and rehabilitative intervention to manage cancer cachexia (PRICC) for patients and their caregivers: protocol for a singlearm feasibility trial. BMJ Open 2021;11:e042883. doi:10.1136/ bmjopen-2020-042883

- Prepublication history for this paper is available online. To view these files, please visit the journal online (http://dx.doi org/10.1136/bmjopen-2020042883).

Received 27 July 2020 Revised 01 February 2021 Accepted 09 February 2021

Deck for updates

(c) Author(s) (or their employer(s)) 2021. Re-use permitted under CC BY-NC. No commercial re-use. See rights and permissions. Published by BMJ.

For numbered affiliations see end of article.

\section{Correspondence to} Dr Loredana Buonaccorso; loredana.buonaccorso@ausl. re.it

\section{ABSTRACT}

Introduction Half of all patients with cancer experience cachexia, with the prevalence rising above $80 \%$ in the last weeks of life. Cancer cachexia (CC) is a complex relational experience that involves the patient-family dyad. There are no studies on the association between the psychoeducational component and the rehabilitative component of dyads for supporting more functional relationships in the management of CC.

The primary objective of this study is to evaluate the feasibility of a psychoeducational intervention combined with a rehabilitative intervention on dyads.

The secondary objective is to improve the quality of life (QoL) and acceptability of the intervention.

Methods and analysis This mixed-methods study with a nonpharmacological interventional prospective includes 30 consecutive cancer patients with cachexia and refractory cachexia and their caregivers, assisted by the Specialised Palliative Care Team. The recruitment will last 1 year. The intervention involves two components: (1) psychoeducational intervention: 3 weekly face-to-face consultations between dyads and trained nurses to help the dyads cope with involuntary weight loss and strengthening dyadic coping resources and (2) rehabilitation intervention: 3 biweekly educational sessions between dyads and trained physiotherapists focused on self-management, goal-setting, physical activity with three home exercise sessions per week. The primary endpoint will be in adherence to the intervention, indicated by a level of completion greater than or equal to $50 \%$ in both components. The secondary endpoints will be QoL (Functional Assessment of AnorexiaCachexia Therapy), caregiver burden (Zarit Burden), physical performance (Hand-Grip strength and 30 seconds sit-to-stand test), and the acceptability of the intervention (ad hoc semi-structured interviews with the dyads and the healthcare professionals).

Ethics and dissemination The study was approved by the Ethics Committee Area Vasta Emilia Nord, Azienda USL-IRCSS Reggio Emilia, Italy, number: 73/2019/SPER/ IRCCSRE. The authors will provide the dissemination of the results through publication in international scientific journals.
Strengths and limitations of this study

A small size and no control group, as in most feasibility studies.

- Innovative bimodal approach, combining psychoeducational and rehabilitative intervention in order to manage cancer cachexia.

- Intervention address to dyads (patients-caregiver) suffering from cancer cachexia and not only to patients.

- Collection of both qualitative and quantitative data due condition of patients involved.

- Training and recurrent supervision of nurses and physiotherapists involved in the intervention.

Trial registration number NCT04153019.

\section{INTRODUCTION}

Approximately half of all patients with cancer experience cachexia, with the prevalence rising above $80 \%$ in the last weeks of life. ${ }^{1-3}$ Cancer cachexia (CC) is a continuum with three stages of clinical relevance: precachexia, cachexia and refractory cachexia. ${ }^{3-5}$ Given the multifaceted pathophysiology and heterogeneous presentation of CC, a personalised treatment approach is necessary to ensure the best possible adherence to the therapeutic proposal, ${ }^{6}$ especially among patients in palliative care, where a $20 \%$ dropout index has been detected. ${ }^{7}$

Core component interventions should, therefore, include nutritional support, as well as exercise-based, anti-inflammatory and educational interventions. ${ }^{3-5}$ However, evidence-based practice indicates that it is difficult for patients to comply with all of these components, with the intake of supplements 
and non-steroid, anti-inflammatory drugs being the most abandoned components. ${ }^{8}$

Exercise can reduce the effects of CC by modulating muscle metabolism, reducing insulin resistance and decreasing the inflammatory cascade. ${ }^{9}{ }^{10}$ Physical activity seems to act as an important anabolic stimulus, especially for patients on chemotherapy, but little is known about its efficacy in the advanced stages of disease. ${ }^{3-5}$

The most recent Cochrane review on this subject examined the safety, acceptability and efficacy of exercise in adult patients with cachexia, but no randomised control trial (RCT) has studied physical activity programmes in patients with advanced diseases. ${ }^{11}$

Cheville et $a l^{12}$ conducted a RCT and investigated the feasibility and impact of a programme of physical activity on the physical well-being of patients with cancer with life expectancy of greater than 6 months who were undergoing radiotherapy. He found that patients in the intervention group experienced subjective improvements in physical performance, but the physical exercise programme did not change their functional status when measured with an objective scale.

Oldervoll $\mathrm{et} \mathrm{al}^{13}$ conducted a RCT to investigate changes in fatigue and physical performance after 8 weeks of physical exercise in patients with cancer with a prognosis of less than 2 years. He did not report any differences with respect to the main outcome, but the intervention group showed a statistically significant improvement in muscle strength, as measured by the grip strength test, and an improvement in physical performance, as measured with the Shuttle walk test.

Solheim conducted a randomised phase II feasibility trial of a multimodal intervention for the management of cachexia in lung and pancreatic cancer, offering nutritional counselling and support, anti-inflammatory drugs and exercise programmes (eg, aerobic and resistance) to patients in the intervention group as opposed to standard care. The intervention was feasible and safe, but recruitment and compliance were suboptimal for exercise. ${ }^{14} \mathrm{~A}$ phase III study is now underway to fully assess the effect of the intervention.

Considering the characteristics of patients with cachexia-anorexia, an exercise programme must be tailored and feasible at home and include resistance exercises as a key component. Some studies have highlighted that resistance exercises stimulate the building of muscle mass and increase strength, thereby reducing the inflammatory response. ${ }^{6-9}$ The authors who have studied this topic proposed an intervention with a duration between 5 and 8 weeks ${ }^{912}$ and a moderate exercise intensity. Other important components of the physical activity programme aimed at motivating the patient and supporting compliance for goal setting, ${ }^{9}{ }^{12}$ stretching and relaxation exercises. ${ }^{15}$

Cachexia-anorexia syndrome also has effects on psychosocial aspects.

Many qualitative studies have shown that CC is associated with psychosocial distress for both patients and their families, with severe consequences on the QoL and on bereavement depression. ${ }^{16-21}$ The psychosocial effects of CC are defined as negative emotions associated with a reduced dietary intake, an involuntary weight loss and social consequences from these symptoms. ${ }^{16}{ }^{17}$

Weight loss generates visible physical changes, creating emotional distress. ${ }^{16} 17$ The patients often do not feel comfortable meeting people because their body image is unfamiliar to them, resulting in an alienation from one's self. $^{1516}$

The involuntary and rapid loss of weight is a cause of concern because it is associated with the fear of imminent death. ${ }^{20}{ }^{21}$ Consequently, some patients strive to eat more, despite the sense of satiety, expressing unrealistic demands of meals to families. The patients often have insight into their clinical condition, and they are more aware of the short-term prognosis than their families. ${ }^{20} 21$

The families undertake a series of practices with the aim of satisfying the nutritional needs of patients, including cooking different foods and spending time buying new foods that can stimulate the appetite of their loved ones. ${ }^{19}$ Many patients feel uncomfortable in refusing food prepared with great care by family members, and consequently, they eat more and more. Alternatively, they choose to close themselves off and socially retreat to avoid further discussion. ${ }^{20}$ Patients who live with their partners report more eating-related distress than patients who live alone. $^{22}$

Studies on psychoeducational approaches to support patients and their families are becoming more common. ${ }^{23-25}$

To our knowledge, there are three studies that provide evidence about the important role of psychosocial interventions in the management of CG. ${ }^{23-25}$

In particular, a mixed-methods qualitative research study $^{25}$ developed a complex family-centred psychosocial intervention to help patients with incurable cancer and their family caregivers to cope with involuntary weight loss and worsening appetite. This intervention was delivered during face-to-face consultations between 16 patientfamily caregiver dyads and a trained nurse. Although 15 dyads reported benefits, the shortcomings must be considered, such as the small sample, the single-centred nature of the intervention and its delivery by alone nurse researcher.

To date, there are no studies on the association between the psychoeducational component and the rehabilitative component of dyads to support more functional relationships for the management of CC. The psychoeducational and rehabilitative components have a strong rationale, common objectives and modalities compared with the multimodal approaches explored in the literature.

We propose a prospective mixed-methods pilot study that assesses the feasibility of a psychoeducational intervention combined with rehabilitation among a population of cancer patients with cachexia and refractory cachexia undergoing care by the Specialised Palliative Care Team (SPCT). 


\section{METHODS AND ANALYSIS}

\section{Aims}

\section{Primary objective}

To evaluate the feasibility of psychoeducational intervention combined with the rehabilitative intervention among dyads to treat CC, assessed through completion rate.

\section{Secondary objective}

- To evaluate the QoL (measured by the Functional Assessment of Anorexia-Cachexia Therapy), caregiver burden (measured by Zarit Burden Scale), upper limb physical performance (measured by HandGrip strength test), lower limb physical performance (measured by 30 seconds sit-to stand test). These evaluations are aimed to collect descriptive data that will be used to establish power calculations required for a full-scale study.

- To evaluate the acceptability of the intervention among dyads (measured by ad hoc semistructured interviews with the dyads and the healthcare professionals).

\section{Sample and setting}

This study includes a convenient sample of 30 consecutive cancer patients with cachexia and refractory cachexia, as well as their caregivers. The study will be conducted in Palliative Care Unit, in collaboration with Physical Medicine and Rehabilitation Unit, of Santa Maria Nuova Hospital, Azienda USL-IRCSS of Reggio Emilia. All patients will be assisted by the SPCT, and the recruitment will last 1 year.

The inclusion criteria are as follows: age greater than 18 years; good command of the Italian language; written consent; histologically confirmed tumour diagnosis; presence of cachexia and refractory cachexia (5, MUST calculation); an identified caregiver by the patient; patients and family members informed of the diagnosis and the objectives of the therapies who have reported awareness of the disease phase (evaluated by the palliative care physician).

The exclusion criteria are as follows: patients with prognosis of less than 3 months; presence of important mental disorder or dementia; severe sensory deficit; presence of diffused bone metastases that put the patient at risk of fracture during exercise.

\section{Intervention}

The intervention includes two components: psychoeducational activities and rehabilitation sessions. The study does not include any restriction of concomitant care or intervention for patients involved. The two nurses and the two physiotherapists involved in the intervention will receive pre-enrolment training and supervision during the study.

\section{Training for nurses and physiotherapists}

The training will include two 4-hour sessions per week for 2 weeks. The main theories on family types will be reviewed with a particular focus on the context of palliative care and the management of the patient/caregiver dyad. $^{26-28}$ The contents of the training will focus on the characteristics and methods of conducting a psychoeducational intervention, specifically related to CC. The theoretical component will be integrated into the practical part through the analysis of clinical cases proposed by nurses and physiotherapists, including the analysis of the functioning of the dyads related to food management. This training will be conducted by the Principal Investigator (PI) (LB), who is a psychologist, psychotherapist, and expert in both palliative care and the support of a functional family system.

\section{Psychoeducational intervention}

The intervention occurs during a face-to-face consultation between a dyad and trained nurses (table 1 shows the timeline of assessment; figure 1 shows the timeline of the

Table 1 The timeline of assessment

\begin{tabular}{|c|c|c|c|c|}
\hline Assessment & $\begin{array}{l}\text { T0 (before } \\
\text { intervention) }\end{array}$ & $\begin{array}{l}\text { T1 ( } 2 \text { weeks } \\
\text { after T0) }\end{array}$ & $\begin{array}{l}\text { T2 ( } 4 \text { weeks } \\
\text { after T0) }\end{array}$ & $\begin{array}{l}\text { T3 (8 weeks } \\
\text { after T0) }\end{array}$ \\
\hline $\begin{array}{l}\text { Functional assessment of Anorexia/Cachexia Therapy } \\
\text { (patient) }\end{array}$ & $\mathrm{x}$ & & $x$ & \\
\hline Zarit Burden Interview (caregiver) & $\mathrm{x}$ & & $x$ & \\
\hline Family Function Index (patient and caregiver) & $\mathrm{x}$ & & & \\
\hline $\begin{array}{l}\text { Number of sessions attended for rehabilitative } \\
\text { intervention }\end{array}$ & & $\mathrm{x}$ & $x$ & $\mathrm{x}$ \\
\hline $\begin{array}{l}\text { Number of sessions attended for psychoeducational } \\
\text { intervention }\end{array}$ & & & $x$ & \\
\hline
\end{tabular}


Identification of eligible dyads and presentation of the study

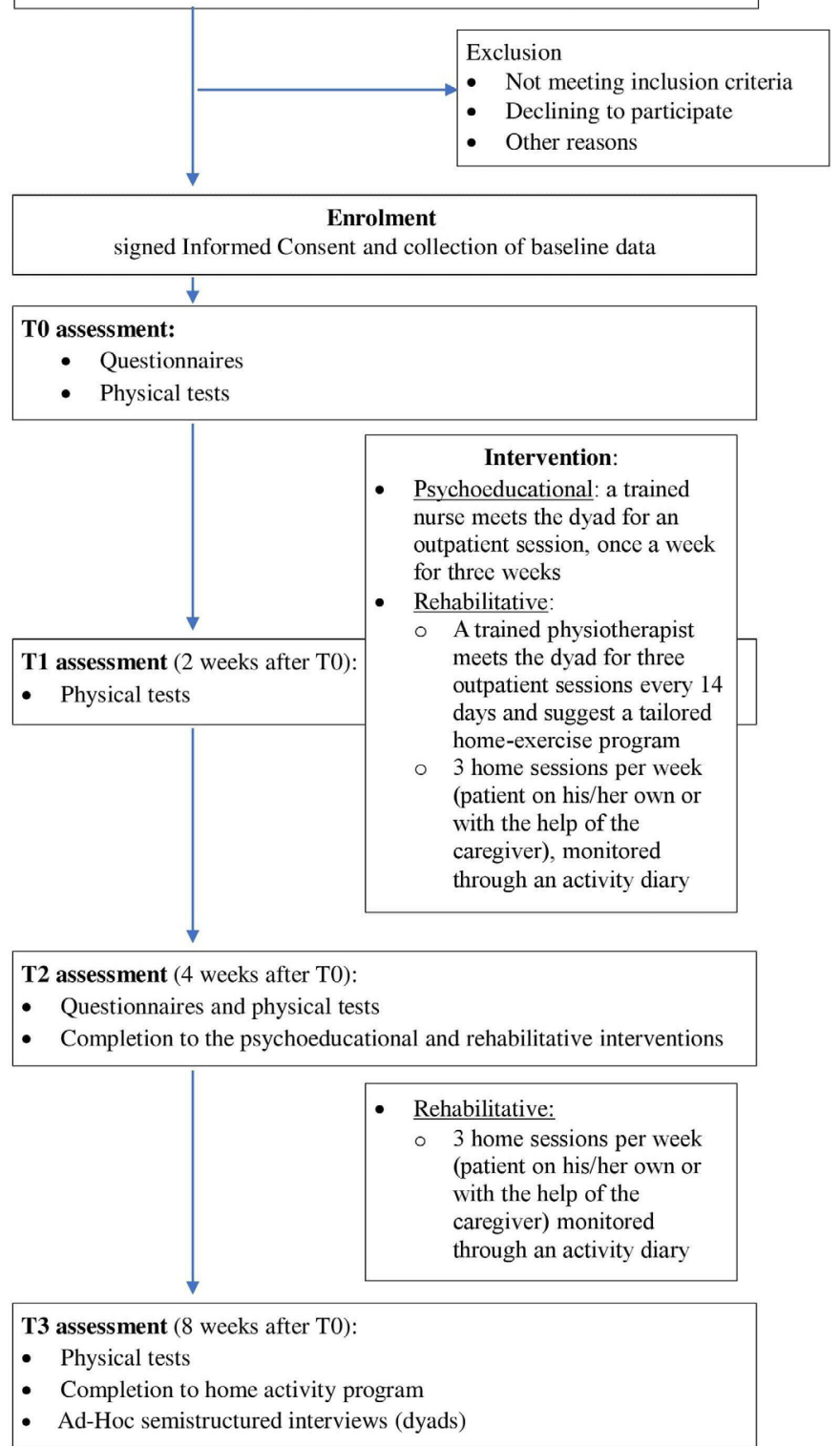

Figure 1 Timeline of the intervention.

intervention). It is designed to help the dyad cope with involuntary weight loss and declining appetite by seeking to strengthen individual and dyadic coping abilities. The intervention includes three meeting, one a week, for outpatients (SPCT) to help the dyads discuss weight and eating-related problems and solutions and to address the following topics consecutively:

1. The 'Mapping changing eating habits' helps the dyads to talk about their own experience of the patient's weight change and eating habits (eg, the role of the family during the preparation of meals, meanings associated with food and reactions of the patient). The nurse should empower the dyad to understand the nature, course, and biological mechanisms of cachexia and acknowledge its negative effects (eg, patients' weight loss, reduced appetite and early satiety). At the end of the meeting, the nurse will record the main needs reported by the dyad with respect to both the management of mealtimes and the experiences associated with them and will ask the dyads to observe their own relational dynamics of the role that food takes during the day. To conduct this meeting and collect data, the nurse will use a tool for evaluating family functioning to construct a first map of the interaction of the dyad. ${ }^{29}$

2. The sharing of data collected during the first meeting will occur among both the nurse and the dyad. The nurse will propose practical examples of different ways of managing food in the care of the patients and propose other ways to support them. In particular, the nurse will facilitate discussion about the other person's perspectives, feelings, and food and provide suggestions for how the patient and family can help each other manage weight-related and eating-related problems. These reflections are embedded in a naturalistic conversation.

3. A final re-evaluation meeting will focus on the needs that will have emerged.

\section{Rehabilitative intervention}

The intervention is conducted by a trained physiotherapist in a face-to-face session with the dyad and includes the following:

1. Three individual outpatient sessions (times T0, T1 and T2; table 1 shows the timeline of assessment; figure 1 shows the timeline of the intervention) will focus on explaining self-management principles and setting goals. The physiotherapist will suggest a tailored physical activity programme to manage or cope with cachexia-related fatigue. The main type of exercise to be proposed is strengthening exercises; the choice of exercise and the number of repetitions will be based on the patient's clinical condition, physical performance and preferences. During each session, the dyad is trained by the physiotherapist to perform the new tailored physical activity programme at home, including the use of weights and/or elastic bands with different strengths, if indicated, as well as the use of stretching and relaxation exercises, if necessary.

2. Three home sessions of exercises per week will be carried out by the patient on his or her own or with the help of the caregiver for a total of minimum 24 sessions over 8 weeks. The patients will keep an activity diary, as previously explained by the physiotherapist, in which they will report the details of the programme done at home (eg, days, type of exercise, duration, repetitions, problems occurred). The caregiver may help patients perform exercises or record in the diary. The dyad is given a booklet in which the key concepts from the face-to-face sessions will be summarised. There is a personal list of exercises determined in collaboration with physiotherapist (ie, for each exercise, there is the figure and explanation) and the Physical Activity diary that the patients are asked to keep for the home exercises. 


\section{Supervision of the entire process}

At the end of each meeting between the nurse/physiotherapist and dyads, the care team will meet with a psychologist/psychotherapist/expert in palliative care and in the family function family system (LB) to discuss the data concerning the dyads' functioning related to CC. The aim will be to modulate the next intervention by considering the needs that emerged among the dyads.

In particular, the bimodal intervention is in continuity with the SPCT, which can be activated according to the needs that have emerged (eg, palliative care physician, psychologist, nutritionist). The palliative care physician will be informed of any information needs about the prognosis or any clinical condition that may emerge during the psychoeducational intervention. If a psychotherapeutic need emerges, especially among patients with refractory cachexia, the psychologists of the PsychoOncology Unit with skills in palliative care will be notified. Team meetings that take place twice a week may provide an additional space for sharing and discussing the global management of patients.

\section{Data collection strategies}

Recruitment will last for 1 year, and all patients assisted by the SPCT will be evaluated during this period. In particular, the palliative care physician (ST) will propose to each dyad meeting inclusion criteria to participate to the study, then an external researcher (GM) will collect written informed consent and baseline evaluation. Each patient will be offered both interventions (psychoeducational and rehabilitative) and will not be given the possibility to choose just one.

All replies and reasons for any refusal to participate will be recorded.

For each participant, the following basic information will be collected immediately after giving informed consent: age, sex, marital status, family unit, education, profession, religious practice, location of the primary tumour, date of diagnosis, and Karnofsky Performance Status. Data will be collected electronically from the Azienda USL-IRCCS of Reggio Emilia database in anonymously and aggregated modalities.

\section{Analysis}

Two external researchers will be involved in the data analysis.

\section{Primary objective}

Completion will be measured as the number of dyads who completed the intervention/the number of involved dyads. Completion will be assessed for each individual component (psychosocial/rehabilitative intervention). The overall intervention will be identified as feasible if there is completion $\geq 50 \%$ for both components.

\section{Secondary objectives}

The effects of the intervention are collected to have descriptive data that will be used to establish power calculations required for a full-scale study.
The effects of the intervention will be evaluated using the following measures:

- QoL and anorexia and cachexia syndrome-related distress will be measured by the Functional Assessment of Anorexia/Cachexia Therapy. ${ }^{30} 31$

- Caregiver burden will be measured by the Zarit Burden Scale. $^{32} 33$

- Upper limb physical performance will be measured by the Hand-Grip strength test. ${ }^{34}$

- Lower limb physical performance will be measured by the 30 seconds sit-to stand test. ${ }^{35}$

- Qualitative secondary aims include the exploration of the acceptability, perceived benefits and concerns, strengths and weaknesses of the intervention from the point of view of interviewed dyads and healthcare professionals.

\section{Measurement scale}

Functional Assessment of Anorexia-Cachexia Therapy

This scale evaluates anorexia and cachexia-related distress. It derives from the FAACT-General and includes four subscales developed and validated to measure health-related QoL, particularly in patients with cancer undergoing therapy: physical well-being, social/family well-being, emotional well-being and functional wellbeing. In addition to the 28 items across these four subscales, 12 specific items were added to assess problems related to the presence of anorexia-cachexia. Response options for reach item ranged from 0 as 'not at all' to 4 as 'very much' with higher scores indicating a greater QoL. The questionnaire can be self-reported or administered by a trained person. ${ }^{30} 31$

In our study, we will use only the subscale related to anorexia-cachexia self-reported by the patient. ${ }^{30}$

\section{Zarit burden scale}

The Zarit Burden Interview is a 22-item instrument for measuring the caregiver's perceived burden of providing family care. The 22 items are assessed on a 5-point Likert scale, ranging from $0=$ 'never' to $4=$ 'nearly always'. Item scores are added to obtain a total score ranging from 0 to 88 , with higher scores indicating greater burden. The questions focus on major areas such as caregiver's health, psychological well-being, finances, social life and the relationship between the caregiver and the patient. ${ }^{32}$

This scale has been widely used for many years and has been translated into 18 languages and validated in Italian. $^{33}$

In this study, it will be self-reported by the caregiver.

\section{Family relationship index}

The self-reported Family Relationship Index is composed of 12 items with true/false answers, and it is specifically used to evaluate family functioning in the palliative care setting. It is used as a screening tool to preventively assess families at risk of developing psychological distress associated with diseases with poor prognoses. Family members report their perceived level of cohesion, expression and 
resolution of conflicts. The scale identifies five family types that are distributed along a continuum ranging from families with good adaptation to the disease (ie, supportive and able to face and resolve conflicts) to dysfunctional families with poor adaptation (ie, hostile and silent, where a high index of anger is detected or latent). There is a defined intermediate family type that is placed between these types of family functioning (eg, with good adaptation and poor adaptation). ${ }^{27}$

In our study, the scale will be used to collect data useful for the evaluation of family functioning, associating them specifically with cachexia and the role of food in assistance (ie, Do we talk about it in the family? Do we tend to avoid the subject? Is it possible to talk about the emotional problems related to cachexia? Is the problem faced or avoided?). The scale will be self-completed separately by the patient and the caregiver.

The data collected will be used by nurses during the psychoeducational intervention as support in the evaluation of the dyad interaction modalities.

There is a validated Italian version. ${ }^{28}$

\section{Hand-Grip strength test}

This test is administered using a dynamometer to quantitatively and objectively measure the isometric muscle strength of the patient's upper limbs. The assessor must calculate the average of three consecutive tests. This test has also been validated for healthy adults, elderly and patients with stroke. It has been used as a measure of physical performance in some studies with patients with cancer, even in advanced stages. ${ }^{34}$

\section{A 30 seconds sit-to-stand test}

This test evaluates the lower limb strength in the sit-tostand function. ${ }^{35}$

The assessor asks the patient to get up from a chair without the support of the arms. The number of repetitions the patient can do in $30 \mathrm{~s}$ is the score of the test.

This test has been validated for patients with osteoarthritic and elderly problems. It has been used as a measure of physical performance in some studies with cancer patients, even in advanced stages.

- Ad hoc semistructured interviews of the dyad will be conducted 1 month after the intervention. The interviews will be conducted by a research medical doctor (GM) trained in qualitative research and focus groups and by the PI of the study (LB) for a total of seven interviews. The dyads will be chosen based on preintervention and postintervention results, with a particular attention to identifying the participants who have reported good adherence to the intervention and participants who have reported difficulties.

- Ad hoc semistructured interviews of nurses and physiotherapists who participated in the study will also be conducted. The interviews will be conducted by a research medical doctor (GM) for a total of four interviews corresponding to the operators involved (two nurses and two physiotherapists).

\section{Data analysis}

The data analysis will be conducted by the Clinical and Statistical Studies Unit Azienda USL-IRCCS of Reggio Emilia.

Sample size: Since this is a pilot study to collect data on the feasibility of the intervention, a formal calculation is not carried out. Thirty patients will be assessed, in accordance with the criteria of opportunity and feasibility. The statistical analysis will be descriptive; the primary endpoint will be represented by descriptive statistics (average, median, minimum, maximum, remarkable percentiles, central tendency index (with 95\% CI), SD and shape indexes).

The secondary endpoint will be the descriptive representation (for T0, T1, T2 and T3) of the endpoints and of the variations detected between $\mathrm{T} 0$ and $\mathrm{T} 3$.

\section{The qualitative analysis}

The qualitative analysis will be performed as described below. Recordings of the interviews will be transcribed verbatim and then analysed using thematic analysis to explore the content and context of responses. ${ }^{36} 37$

Each transcript will be independently labelled by two researchers, who will resolve differences in labelling. Throughout an iterative process, they will inductively identify a few subthemes. Finally, a third researcher will revise both the transcripts and the preliminary thematic analysis, regroup, and rename some themes and subthemes with the objective of describing them by highlighting commonalities and differences between the perspectives of the three 'actors' involved. This revision will be discussed and amended with the other researchers involved in the qualitative analysis.

\section{Patient and public involvement}

No patients or association were involved in the design or conduction of the study.

However, the development of psychoeducationl and rehabilitative intervention to manage CC intervention was informed by literature research and patients' priorities collected by the SPCT during the clinical practice.

\section{ETHICS AND DISSEMINATION}

The study was approved by the Ethics Committee Area Vasta Emilia Nord, Azienda USL-IRCSS of Reggio Emilia, Italy, with the number 73/2019/SPER/IRCCSRE.

All study participants will be informed in detail of the aims and objectives of the study by the PI (LB) and SPCT investigator and must sign a consent form for the study and processing of personal data that will be filed together with the study documentation. Consent to participate in the study will be requested and collected by the proposing doctor or by the researcher in charge of the T0 assessment. Each participant has the right to withdraw their membership in the study at any time. 
All data collected are restricted by Ethics Committee of the Vast Northern Emilia (Italy) in order to protect participant privacy. The data will be available from the corresponding author (LB), on reasonable request, with the permission of Azienda USL-IRCCS of Reggio Emilia, Italy.

The study will be conducted in accordance with this protocol along with any amendments introduced and authorised, as well as the ethical principles of the Declaration of Helsinki.

The authors will provide the dissemination of the study results through publication in international scientific journals.

\author{
Author affiliations \\ ${ }^{1}$ Psycho-Oncology Unit, Azienda USL - IRCCS di Reggio Emilia, Reggio Emilia, Italy \\ ${ }^{2}$ Palliative Care Unit, Azienda USL - IRCCS di Reggio Emilia, Reggio Emilia, Italy \\ ${ }^{3}$ Physical Medicine and Rehabilitation Unit, Azienda USL - IRCCS di Reggio Emilia, \\ Reggio Emilia, Italy \\ ${ }^{4}$ Department of Medicine, Surgery, Dentistry and Morphological Sciences, University \\ of Modena and Reggio Emilia, Modena, Italy \\ ${ }^{5}$ Scientific Directorate, Azienda USL - IRCCS di Reggio Emilia, Reggio Emilia, Italy \\ ${ }^{6}$ Clinical and Experimental Medicine Ph.D. Program, University of Modena and \\ Reggio Emilia, Modena, Italy
}

Acknowledgements The authors are grateful to Linette N Leng, University of lowa, for the English language editing.

Contributors LB analysed the literature on cancer cachexia, devised the study, coordinated the working of team. She contributed to the conception of the study, to the draft of the manuscript and to its critical revision for important intellectual content, supporting each member of the team. She conducted the healthcare professionals training and handled the presentation of the protocol to the Ethics Committee. She approved the final version to be submitted and agrees to be accountable for all aspects of the work in ensuring that questions related to the accuracy or integrity of any part of it are appropriately investigated and resolved. EB contributed to the design and drafting of the study protocol and the implementation of the psychoeducational intervention. She also collaborated in the writing and rereading of the manuscript. CA contributed to the design and drafting of the study protocol and the implementation of the psychoeducational intervention. She also collaborated in the writing and rereading of the manuscript. MAA contributed to the design and drafting of the study protocol after the analysis of the literature on cachexia related fatigue. She also contributed to the implementation of the rehabilitation intervention and she collaborated in the writing and rereading of the manuscript. MD contributed to the design of the study protocol after the analysis of the literature on cachexia related fatigue. She also contributed to the implementation of the rehabilitation intervention. She also collaborated in the drafting and rereading of the manuscript. SF contributed to the research project development and to the rehabilitation protocol, after the analysis of the literature on cachexia related fatigue. She also collaborated in the drafting and rereading of the manuscript. GM gave a methodological contribution, in particular on qualitative methods. SC contributed to the conception of the study, to the draft of the manuscript and to its critical revision for important intellectual content. ST analysed the literature on cancer cachexia. She contributed to the conception of the study, to the draft of the manuscript and to its critical revision for important intellectual content. She coordinates the patient enrolment in the study as a head of the Specialised Palliative Care Team. All authors read and approved the final manuscript.

Funding This work was supported by Azienda USL-IRCCS of Reggio Emilia, within the Call for Oncological Research Value 2018 (5 per Mille 2016 Funds)-Bando per la Valorizzazione della Ricerca Istituzionale in ambito oncologico 2018 (Fondi 5 per Mille 2016), was selected throughout a competitive evaluation process which involved external referees. The remaining costs were covered by donations to the Scientific Directorate for research activities.

Disclaimer Funders had no role in the definition of the study design, in the collection, analysis or interpretation of data, in the writing of the report or in the decision to submit the article for publication.

Competing interests None declared.
Patient and public involvement Patients and/or the public were not involved in the design, or conduct, or reporting, or dissemination plans of this research.

Patient consent for publication Not required.

Provenance and peer review Not commissioned; externally peer reviewed.

Open access This is an open access article distributed in accordance with the Creative Commons Attribution Non Commercial (CC BY-NC 4.0) license, which permits others to distribute, remix, adapt, build upon this work non-commercially, and license their derivative works on different terms, provided the original work is properly cited, appropriate credit is given, any changes made indicated, and the use is non-commercial. See: http://creativecommons.org/licenses/by-nc/4.0/.

ORCID iDs

Loredana Buonaccorso http://orcid.org/0000-0002-3627-8691

Monica Denti http://orcid.org/0000-0001-6541-2696

Gianfranco Martucci http://orcid.org/0000-0001-6153-5525

Stefania Costi http://orcid.org/0000-0002-4645-0336

\section{REFERENCES}

1 Hopkinson JB, Wright DNM, McDonald JW, et al. The prevalence of concern about weight loss and change in eating habits in people with advanced cancer. J Pain Symptom Manage 2006;32:322-31.

2 von Haehling S, Anker SD. Cachexia as a major underestimated and unmet medical need: facts and numbers. J Cachexia Sarcopenia Muscle 2010;1:1-5.

3 Arends J, Bachmann P, Baracos V, et al. ESPEN guidelines on nutrition in cancer patients. Clin Nutr 2017;36:11-48.

4 Cederholm T, Barazzoni R, Austin P, et al. ESPEN guidelines on definitions and terminology of clinical nutrition. Clin Nutr 2017;36:49-64.

5 Fearon K, Strasser F, Anker SD, et al. Definition and classification of cancer cachexia: an international consensus. Lancet Oncol 2011;12:489-95.

6 Maddocks M, Hopkinson J, Conibear J, et al. Practical multimodal care for cancer cachexia. Curr Opin Support Palliat Care 2016;10:298-305.

7 Hui D, Dev R, Bruera E. The last days of life: symptom burden and impact on nutrition and hydration in cancer patients. Curr Opin Support Palliat Care 2015;9:346-54.

8 Bruera E, Hui D. Palliative care research: lessons learned by our team over the last 25 years. Palliat Med 2013;27:939-51.

9 Maddocks M, Murton AJ, Wilcock A. Therapeutic exercise in cancer cachexia. Crit Rev Oncog 2012;17:285-92.

10 Maddocks M, Jones LW, Wilcock A. Immunological and hormonal effects of exercise: implications for cancer cachexia. Curr Opin Support Palliat Care 2013;7:376-82.

11 Grande AJ, Silva V, Riera R, et al. Exercise for cancer cachexia in adults. Cochrane Database Syst Rev 2014:CD010804.

12 Cheville AL, Girardi J, Clark MM, et al. Therapeutic exercise during outpatient radiation therapy for advanced cancer: feasibility and impact on physical well-being. Am J Phys Med Rehabil 2010;89:611-9.

13 Oldervoll LM, Loge JH, Lydersen S, et al. Physical exercise for cancer patients with advanced disease: a randomized controlled trial. Oncologist 2011;16:1649-57.

14 Solheim TS, Laird BJA, Balstad TR, et al. A randomized phase II feasibility trial of a multimodal intervention for the management of cachexia in lung and pancreatic cancer. J Cachexia Sarcopenia Muscle 2017;8:778-88.

15 Hagmann C, Cramer A, Kestenbaum A, et al. Evidence-based palliative care approaches to non-pain physical symptom management in cancer patients. Semin Oncol Nurs 2018;34:227-40.

16 Reid J, McKenna H, Fitzsimons D, et al. The experience of cancer cachexia: a qualitative study of advanced cancer patients and their family members. Int J Nurs Stud 2009;46:606-16.

17 Amano K, Maeda I, Morita T, et al. Eating-related distress and need for nutritional support of families of advanced cancer patients: a nationwide survey of bereaved family members. J Cachexia Sarcopenia Muscle 2016;7:527-34.

18 Reid J, McKenna H, Fitzsimons D, et al. Fighting over food: patient and family understanding of cancer cachexia. Oncol Nurs Forum 2009;36:439-45.

19 Oberholzer R, Hopkinson JB, Baumann K, et al. Psychosocial effects of cancer cachexia: a systematic literature search and qualitative analysis. J Pain Symptom Manage 2013;46:77-95. 
20 Hopkinson JB. Psychosocial impact of cancer cachexia. J Cachexia Sarcopenia Muscle 2014;5:89-94.

21 Reid J, McKenna HP, Fitzsimons D, et al. An exploration of the experience of cancer cachexia: what patients and their families want from healthcare professionals. Eur J Cancer Care 2010;19:682-9.

22 Lövgren M, Tishelman C, Sprangers M, et al. Symptoms and problems with functioning among women and men with inoperable lung cancer--a longitudinal study. Lung Cancer 2008;60:113-24.

23 Hopkinson JB, Fenlon DR, Okamoto I, et al. The deliverability, acceptability, and perceived effect of the Macmillan approach to weight loss and eating difficulties: a phase II, cluster-randomized, exploratory trial of a psychosocial intervention for weight- and eating-related distress in people with advanced cancer. J Pain Symptom Manage 2010;40:684-95.

24 Hopkinson JB, Fenlon DR, Foster CL. Outcomes of a nurse-delivered psychosocial intervention for weight- and eating-related distress in family carers of patients with advanced cancer. Int J Palliat Nurs 2013;19:116-23.

25 Hopkinson JB, Richardson A. A mixed-methods qualitative research study to develop a complex intervention for weight loss and anorexia in advanced cancer: the family approach to weight and eating. Palliat Med 2015;29:164-76.

26 Kissane DW, Zaider TI, Li Y, et al. Randomized controlled trial of family therapy in advanced cancer continued into bereavement. $J$ Clin Oncol 2016;34:1921-7.

27 Schuler TA, Zaider TI, Li Y, et al. Typology of perceived family functioning in an American sample of patients with advanced cancer. J Pain Symptom Manage 2014;48:281-8.
28 Kissane DW. The challenge of family-centered care in palliative medicine. Ann Palliat Med 2016;5:319-21.

29 Margola D, Fenaroli V S, et al. The family relationships index (FRI): multilevel confirmatory factor analysis in an Italian community sample. Eur J Psychol Assess 2019;35:335-45.

30 Ribaudo JM, Cella D, Hahn EA, et al. Re-validation and shortening of the functional assessment of anorexia/cachexia therapy (FAACT) questionnaire. Qual Life Res 2000;9:1137-46.

31 The quality of life and nutrition in the patient with cancer by William Small J, M.D.; Robert Carrara, R.D.; Lynn Danford, M.S., L.D.; Jeri A. Logemann, Ph.D.; and David Cella, Ph.D. ACCC's Integrating nutrition into your cancer program 2002:15-16.

32 Zarit SH, Reever KE, Bach-Peterson J. Relatives of the impaired elderly: correlates of feelings of burden. Gerontologist 1980;20:649-55.

33 Chattat R, Cortesi V, Izzicupo F, et al. The Italian version of the Zarit burden interview: a validation study. Int Psychogeriatr 2011;23:797-805.

34 Shirley Ryan AbilityLab. Hand-held dynamometer/grip strength., 2014. Available: https://www.sralab.org/rehabilitation-measures/ hand-held-dynamometergrip-strength [Accessed 22 Jan 2019].

35 Shirley Ryan AbilityLab. 30 second sit to stand test, 2013. Available: https://www.sralab.org/rehabilitation-measures/30-second-sit-standtest [Accessed 22 Jan 2019].

36 Taylor M. Interviewing. In: Holloway I, ed. Qualitative research in health care. Maidenhead, England: McGraw-Hill Education, 2005: 39-55.

37 Braun V, Clarke V. Using thematic analysis in psychology. Qual Res Psychol 2006;3:77-101. 